SL Elizaveta Khachaturyan (ed.) Italiano e norvegese: studi di lingua e di cultura, Oslo Studies in Language 10(1), 2018. 67-90. (ISSN 1890-9639)

http://www. journals.uio.no/osla

\title{
PROBLEMI DI TRADUZIONE DI DUE ROMANZI ITALIANI IN NORVEGESE: TRA LA LINGUA E IL DIALETTO
}

PERNILLE THULL Università di Oslo

Sapienza Università di Roma

\section{ABSTRACT}

This article linguistically analyzes how the Sicilian dialect is used in conjunction with Italian in the books Il cane di terracotta (The Terracotta Dog) by Andrea Camilleri and La Mennulara (The Almond Picker) by Simonetta Agnello Hornby. The analysis considers how the dialect is represented at various levels: phonetic, morphological, syntactic, and lexical. In addition, the Norwegian translations of these books are also analyzed: Terrakottahunden, translated by Jon Rognlien, and Mandelplukkersken by Tommy Watz, respectively. It focuses on how the translators solved the challenge of the original books' text alternating between Italian and Sicilian dialect. The translators' account of their choices is presented following each analysis.

Il problema della traduzione di varietà linguistiche non standardizzate come dialetti, socioletti o idioletti, è molto discussa sia dai linguisti sia dai traduttori (ad esempio Eco 2007 e Bellos 2011). Obiettivo di questo articolo è quello di mostrare quanto la diversa situazione sociolinguistica tra la Norvegia e l'Italia possa porre problemi ai traduttori che si confrontano con testi nei quali si mischiano svariati aspetti sociolinguistici. Apparentemente, il traduttore norvegese non dovrebbe avere difficoltà nel tradurre un romanzo italiano ricco di dialetti, perché anche il norvegese è una lingua con un'ampia varietà diatopica; si potrebbe eventualmente anche essere tentati di ricorrere a danese, svedese e norvegese assieme, lingue molto affini tra di loro. Studiando due casi concreti, cercheremo invece di mostrare la difficoltà, e in alcuni casi l'impossibilità, di rendere la funzione di stereotipo dei dialetti italiani in norvegese. Vedremo come due traduttori illustri hanno affrontato questa problematica e quali risultati hanno raggiunto. Non ci sono studi riguardanti l'analisi delle traduzioni dall'italiano al norvegese, almeno per quello che abbiamo potuto constatare finora.

È opportuno ricordare che a causa di avvenimenti storici diversi, il rapporto con la/le lingue standard e l'uso del dialetto in questi due paesi è diverso. L'italiano standard si utilizza prevalentemente nello scritto. Nell'Italia di oggi, il parlante 
è di norma bilingue e nella loro forma orale, la lingua e il dialetto vengono usati quotidianamente separati o insieme per scopi diversi e con interlocutori diversi. ${ }^{1}$ Dunque, nella situazione linguistica odierna, fenomeni come code switching, code mixing e ibridazione (di cui parleremo) caratterizzano la lingua parlata di un italiano medio. In Norvegia la situazione linguistica è diversa: il dialetto è usato nelle situazioni sia informali che formali ed è perfettamente accettato. In generale, ognuno parla il proprio dialetto e i fenomeni di alternanza di codice tra il dialetto e le lingue standard sono piuttosto limitati, mentre l'uso della lingua standard porta con sé una connotazione formale e rigida. Siccome ci sono molteplici dialetti, e la differenza tra essi è notevole, i norvegesi spesso si trovano a fare conversazioni poli-dialettali, in cui l'interazione si svolge in due o più codici diversi. ${ }^{2}$

Questo articolo si propone di presentare un'analisi del modo in cui due traduttori norvegesi hanno affrontato, sebbene in maniera diversa, il problema dell'alternanza di due codici linguistici, il dialetto siciliano e l'italiano. I romanzi che sono oggetto della nostra ricerca sono Il cane di terracotta di Andrea Camilleri e la sua traduzione norvegese Terrakottahunden di Jon Rognlien; e il romanzo La Mennulara di Simonetta Agnello Hornby e la sua traduzione norvegese Mandelplukkersken di Tommy Watz.

La nostra analisi ha due obiettivi. In primo luogo, per capire la strategia dei due scrittori sarà descritto l'uso del dialetto nei due romanzi. In secondo luogo, analizzeremo le scelte traduttologiche dei due traduttori norvegesi, descrivendo come hanno affrontato il problema della presenza di una mutazione del codice linguistico tra il dialetto siciliano e l'italiano nel testo originale.

L'articolo è organizzato nel modo seguente: all'inizio daremo una breve presentazione biografica degli scrittori, con particolare attenzione alla loro relazione con il dialetto siciliano e la lingua standard italiana; in seguito presenteremo due interviste sull'uso del dialetto nelle loro opere, e commenteremo le loro risposte (§1); seguirà un'analisi delle traduzioni norvegesi, iniziando con una riflessione sui problemi teorici associati alla resa delle varietà linguistiche non standardizzate (\$2); per finire, nel paragrafo (\$3), ci occuperemo delle scelte traduttologiche e delle loro giustificazioni da parte dei due traduttori Jon Rognlien e Tommy Watz.

[1] "Nella situazione italiana, in cui sono compresenti lingua e dialetti, il parlante è di norma bilingue, cioè nella vita di tutti i giorni usa sia la lingua che il dialetto, separatamente o insieme, spesso - ma non sempre - utilizzando i due codici per scopi differenti, con interlocutori diversi." (Grassi, Sobrero \& Telmon 2012: 177)

[2] "Swiss standard German and Nynorsk today are used exclusively in (non- private) writing and very formal oral situations. In formal and semi- formal situations, everybody speaks his or her dialect; and since the diversity of the local dialects is enormous (both in Norway and in Switzerland), and modern Norwegians or Swiss are as mobile as any other Europeans, face-to-face interaction is often 'polylectal'. [...]. Some dialects carry more prestige than others, but speaking dialect as such is highly appreciated, while the standard variety has the connotations of a formality and stiffness. Since the written/spoken distinction is so important, the occasions for conversational code- switching between standard and dialect are relatively restricted (i.e. to those situations in which the standard can be used in oral communication at all)." (Auer 2005: 15) 


\section{[1] INTRODUZIONE}

Numerosi sono gli studi sulla lingua di Camilleri. Parallelamente agli studi sociolinguistici (Cerrato 2012) e a quelli traduttologici in altre lingue (Gutkowski 2009), si trovano numerose interviste allo stesso Camilleri nelle quali gli vengono poste domande che riguardano appunto il suo peculiare linguaggio (Sorgi 2000). Lo stile camilleriano rappresenta una sfida per il traduttore non solo per la presenza del dialetto siciliano, ma soprattutto perché fenomeni come code switching, code mixing e ibridazione, sono usati frequentemente. Nei suoi romanzi possiamo trovare quasi tutto il repertorio stilistico dell'italiano che include aspetti diatopici, diafasici, diastratici e diamesici. Non a caso Stephen Sartarelli, ${ }^{3}$ il traduttore inglese di Camilleri, parla di linguistic patchwork and invention e linguistic mosaic.

La lingua della Agnello Hornby, per converso, non è stata analizzata da linguisti o commentata dai traduttori quanto quella camilleriana, probabilmente perché non presenta una varietà linguistica così ampia come la scrittura di Camilleri. Troviamo una varietà diatopica, per la presenza del dialetto siciliano; una varietà diastratica perché i vari modi di parlare dei personaggi manifestano la loro provenienza sociale; e anche una varietà diamesica, legata alla presenza ricorrente di lettere scritte e dialoghi. Ma è soprattutto al livello sintattico che si nota la dialettalità.

\section{[1.1] Terminologia, metodo e approccio}

La terminologia sociolinguistica che useremo per l'analisi si basa prevalentemente sui lavori di Marazzini (2004), Berruto (2012) e Grassi, Sobrero \& Telmon (2012). Il nostro spoglio si basa su un corpus creato in maniera manuale. L'uso del dialetto avviene su più livelli: fonetico, morfologico, sintattico e lessicale.

Per quanto riguarda l'analisi delle strategie adottate per tradurre Camilleri e Agnello Hornby in norvegese, saranno selezionati brani interessanti che mostrano l'approccio particolare dei due traduttori. Il testo originale italiano sarà confrontato con la traduzione norvegese.

Una risposta univoca su come tradurre le varietà linguistiche non standardizzate non esiste. Per avere un'idea riguardo alla prassi più seguita, faremo una breve riflessione sui problemi che si riscontrano nella traduzione di un libro scritto in lingua standard in cui sono presenti varietà linguistiche non standardizzate. Ci baseremo principalmente sugli studi teorici di Eco (2007), Nergaard (2009), Federici (2011) e Bellos (2011). Per giustificare le scelte traduttologiche personali di ciascun traduttore (Jon Rognlien e Tommy Watz), verranno presentate le loro motivazioni, spiegate in due interviste che abbiamo realizzato nel 2013 (separatamente con ogni traduttore). ${ }^{4}$

[3] In Gutkowski (2009: 8-9)

[4] Jon Rognlien è stato intervistato su Skype in italiano, mentre Tommy Watz ha risposto per iscritto, in norvegese, a una serie di domande che gli abbiamo sottoposto. 
[2] ANALISI DEL TESTO ORIGINALE

[2.1] Andrea Camilleri e l'uso del dialetto nel romanzo "Il cane di terracotta"

Andrea Camilleri è nato in Sicilia a Porto Empedocle nel 1925. Da giovane si trasferì per gli studi a Roma dove vive a tutt'oggi. Oltre ad essere scrittore è stato innanzitutto un regista di teatro, della radio e della televisione. È nel 1994 che con il primo libro della serie di Montalbano, La forma dell'acqua, iniziò il suo vero successo. Oggi Camilleri è uno scrittore di grande fama sia nazionale che internazionale. Il romanzo scelto per questa analisi è Il cane di terracotta, appartenente alla serie di Montalbano.

Durante l'infanzia in Sicilia, Camilleri parlava principalmente in dialetto e solo molto tempo dopo ha cominciato a parlare anche l'italiano. ${ }^{5}$ Trasferitosi a Roma, costretto a parlare la lingua nazionale, lo scrittore si è trovato in difficoltà e si è reso conto che in realtà mentre parlava traduceva dal siciliano, come ricorda egli stesso: "Una traduzione di necessità limitativa, e dire che l'italiano lo conoscevo bene, ma rispetto a come conosco il siciliano, era come parlare in inglese. Certe sfumature, certe cose mi sfuggivano". ${ }^{6}$ Anche all'inizio della sua produzione letteraria, Camilleri ha avuto difficoltà a trovare uno stile personale che gli permettesse di sentirsi a suo agio.

Dai dati che ricaviamo da un'intervista che abbiamo effettuato a Roma nel 2013 presso la sua abitazione, possiamo osservare come Camilleri si senta più vicino al dialetto e quanto gli venga più facile adoperarlo. Del resto, alla nostra domanda: "Perché usa il dialetto nella sua scrittura?", egli ci ha dato una risposta che rispecchia la definizione di code switching:

"Perché adoperiamo il dialetto e la lingua, noi, in due modi diversi? Allora, la cosa che racconto sempre io è il ricordo di un discorso che mi fece mia madre, quando io avevo sedici anni, diciassette anni, e mi diede la chiave della casa. Ed io ho cominciato a tornare alle due, alle tre di notte. Un giorno mia madre si scocciò e mentre eravamo a tavola, mi disse: 'Nene, figlio mio, cerca di tornare presto la notte, perché se io non sento la porta ca si chiuca', che significa che tornassi, 'non arrinèscio a pigliare sogno e perdo la nottata. E se questa storia dura, io non ti do più una lira, e voglio vedere che cosa fai di notte fino alle tre'. Allora, tutta la prima parte del discorso di mia madre era in dialetto ed era una mozione degli affetti, un discorso affettivo. La seconda parte che era poliziesca, intimidatoria, notarile, era fatta in italiano". ${ }^{7}$

[5] L'intervista integrale si trova in: http://www.letteratura.rai.it/articoli-programma/ linvestigatore-ecamilleri/1193/default.aspx.

[6] Scrittorincorso. Linguaggio 2013

[7] Intervista personale trascritta da me, Roma 19.02.2013. 
Nella stessa intervista, Camilleri ricorda anche una celebre frase di Pirandello in cui egli dice: "di una data cosa, il dialetto ne esprime il sentimento, della medesima cosa la lingua ne esprime il concetto".

La difficoltà di tradurre il linguaggio camilleriano è sicuramente legata alla creatività da parte dell'autore nel mischiare il dialetto con la lingua nazionale e nel fatto di esplorare tutto il repertorio linguistico italiano per creare il modo unico di parlare di ciascun personaggio. Ne Il cane di terracotta l'uso del dialetto svolge un ruolo eminente e, assieme alla vasta presenza di dialoghi, la lingua conferisce un'impronta di oralità al testo. La lingua presentata nel libro corrisponde a un siciliano medio con tratti sud-occidentali, e appare ovunque. Tuttavia esso non viene usato in un modo univoco. Del resto i romanzi di Camilleri non appartengono alla letteratura dialettale in senso stretto perché il dialetto non viene usato in modo costante. Ci sono alcune descrizioni nelle quali appare un'ampia quantità di parole siciliane e allo stesso tempo descrizioni nelle quali il dialetto viene usato meno. La stessa cosa vale per i dialoghi, alcuni dei quali si svolgono in dialetto siciliano, altri in un italiano privo di caratteristiche regionali, popolari o colloquiali e alcuni in una combinazione di dialetto e italiano. La lingua camilleriana non rappresenta solo un intreccio tra l'italiano standard e il dialetto siciliano, ma, considerando i dialoghi, quasi tutto il repertorio stilistico dell'italiano viene utilizzato. Generalmente ci sono poche descrizioni di paesaggi, di ambiente, di personaggi, ecc. A tal riguardo, Cerrato (2012: 24) afferma che Camilleri "presenta i personaggi attraverso il loro stesso linguaggio e non attraverso un profilo descrittivo e introduttivo, quindi ogni personaggio del libro ha un suo modo di parlare, sapientemente creato dal narratore". La dialettalità è presente a tutti i livelli linguistici: fonetico, morfologico, sintattico e lessicale.

\section{[2.2] Simonetta Agnello Hornby e l'uso del dialetto nel romanzo "La mennulara"}

Il secondo romanzo analizzato, La Mennulara scritto da Simonetta Agnello Hornby, è stato scelto per i seguenti motivi: Agnello Hornby, come Camilleri, è una scrittrice contemporanea, usa il dialetto siciliano nelle sue opere ed è stata tradotta in norvegese.

Simonetta Agnello Hornby nasce a Palermo nel 1945. Laureata in giurisprudenza nel 1967, si trasferisce all'estero, vive negli Stati Uniti e in Zambia, poi nel 1970 si trasferisce a Londra. Qui apre nel 1979 lo studio legale Hornby and Levy che si specializza ben presto in diritto familiare e minorile. Ancora oggi lavora come avvocato per questo genere di cause e per lo stesso studio legale. Nel 2002 debutta come scrittrice con il romanzo La Mennulara, pubblicato da Feltrinelli. ${ }^{8}$

Alle domande della nostra intervista sul suo rapporto con il dialetto siciliano e con l'italiano e sulla sua infanzia, la scrittrice risponde che quando era piccola a casa si parlavano entrambe le lingue. Non avendo frequentato la scuola prima

[8] http://www.agnellohornby.it/biografia/ 
dell'età di dieci anni, ebbe una maestra privata ogni mattina a casa con la quale parlava solo ed esclusivamente in italiano. La Agnello Hornby parlava in italiano anche con sua madre, la quale non era a proprio agio con il dialetto siciliano. Capiva e usava in famiglia parole siciliane nelle conversazioni quotidiane, però evitava di esprimersi esclusivamente in dialetto. Il padre, per conto suo, era abile a esprimersi in ambedue codici. La Agnello Hornby riferisce che da piccola parlava con le persone di servizio, con i portieri e con l'autista, nonché con i contadini e i loro figli esclusivamente il dialetto. Alla domanda se è capace di utilizzare in tutti i contesti il dialetto e l'italiano, la scrittrice risponde che si considera "bilingue" nel parlato ma non nello scritto. Lei si ritiene solo capace di scrivere in italiano, perché il siciliano non è stato riconosciuto come lingua scritta. Il dialetto non è la sua prima lingua, come per Camilleri. ${ }^{9}$ Alla nostra domanda sul perché lei usi il dialetto nei suoi romanzi, la risposta della Agnello Hornby è stata:

"Uso il siciliano quando non trovo una parola italiana che descrive quello che voglio. Uso la struttura siciliana sintattica quando faccio parlare gente che normalmente non parla italiano. Uso il siciliano quando si parla di affetti, per esempio dico nico anziché "piccolo piccolo', è più dolce e mia madre lo usava".

In un'altra intervista con l'Accademia Apulia UK, la scrittrice racconta:

“[...] non uso parole in siciliano per 'arricchire' il racconto, ma solo perché non conosco il corrispondente italiano. È un problema d'ignoranza. Per esempio uso spesso la parola taliata, che significa più o meno 'guardare', perché non so come rendere in italiano il significato che io credo che questa abbia". ${ }^{10}$

Tale affermazione è molto interessante perché, come successivamente descritto nell'analisi linguistica, le poche parole in dialetto vengono usate spesso nei casi in cui non c'è un equivalente nella lingua italiana. Del resto, $i$ brani descrittivi usano esclusivamente la sintassi italiana, mentre i dialoghi tra i personaggi, soprattutto se appartenenti a un ceto sociale basso, seguono più volentieri la sintassi dialettale.

\section{[2.3] Il dialetto in Camilleri e nella Agnello Hornby}

Paragonando i due romanzi, si nota come Camilleri usa di più il dialetto a tutti i livelli. Il libro della Agnello Hornby invece, presenta uno spettro piuttosto limitato di tratti dialettali.

[9] La scrittrice ha risposto per iscritto alle domande che le avevamo inviato (il 04.06.2013).

[10] L'intera intervista si trova in: http : / / www . accademiapulia . org / it / members / simonetta agnellohornby-avvocato-scrittrice-244.html 
Come esempio di cambiamento fonetico possiamo citare la metafonia,fenomeno che consiste nella chiusura della vocale per influsso di vocali atone come $[\mathrm{i}] \mathrm{e}[\mathrm{u}]$ nella sillaba seguente, qui rappresentato dal mutamento [e] > [i] in fimmina per femmina e in virità per verità. Camilleri scrive milanzane invece di melanzane come sarebbe in italiano standard. La lingua di Camilleri è fortemente caratterizzata da varie particolarità fonetico-grafiche, per esempio la parola siciliana iurnu per "giorno" viene scritta jornu. Jornu è un ibridismo perché abbiamo la o nel mezzo della parola italiana, mentre la vocale finale è la tipica $u$ siciliana. Vediamo anche che Camilleri non inizia la parola né con la $i$ come in siciliano, né con la $g$ come in italiano, invece con la $\mathrm{j}$. Al contrario la parola iurnata per "giornata" viene scritta nella sua forma siciliana propria. Il fenomeno di ibridizzazione non è presente nel libro della Agnello Hornby, in cui non ci sono esempi d'ibridismi, giacché le parole sono scritte o in italiano o in siciliano. Quando scrive in dialetto usa dialettismi, ai quali è difficile trovare degli equivalenti italiani. Per esempio, la parola siciliana passio appare più volte nel testo e vuol dire letteralmente "passeggio" dal verbo "passeggiare". Ma nel dialetto siciliano la forma il passì indica l'usanza di passeggiare per la piazza o per le vie del paese. ${ }^{11}$ Quindi osservare il passio vuol dire "osservare la gente che passeggia". La parola appare nel testo senza una concreta spiegazione e senza essere inserita tra virgolette, come si può vedere nel seguente esempio:

"Il pomeriggio del 23 settembre don Vito era seduto nella loggia da cui controllava contemporaneamente l'ingresso, il passìo in piazza e le attività della sua famiglia all'interno della portineria". ${ }^{12}$

Come esempio di cambiamento fonetico possiamo citare la parola "cervello" che in dialetto siciliano diventa "ciriveddu", ma che nel romanzo Camilleri scrive sempre "ciriveddro", aggiungendo una $r$ prima della vocale finale e sostituendo la $u$ finale siciliana con la o italiana.

Per quanto riguarda il livello sintattico, nel romanzo di Camilleri la sintassi segue prevalentemente quella italiana, con alcune eccezioni. I brani raccontati dalla voce narrante seguono per la maggior parte la struttura di una frase italiana, mentre i dialoghi - usano la struttura dialettale. Per esempio, una caratteristica nota del dialetto siciliano è "la collocazione dei verbi, in particolare delle forme di 'essere' ed 'avere', alla fine della frase". ${ }^{13}$ In Camilleri, un esempio di questo tipo di inversione è la famosa frase: Montalbano sono. Altri esempi: Bene sto; ${ }^{14}$ Pronto?

[11] Informazione ottenuta in una comunicazione personale con un siciliano. Il lemma è assente nei dizionari consultati.

[12] La Mennulara: 28

[13] Tropea (1976: 45)

[14] Il cane di terracotta: 12 
Pronto? Montalbano? Salvuzzo! Io sono, Gegè sono, ${ }^{15}$ e Dal punto di vista della curiosità, fimmina certamente non era. ${ }^{16}$

Nella Agnello Hornby è a livello sintattico che si nota di più la dialettalità. Come in Camilleri, le descrizioni seguono esclusivamente la sintassi italiana, mentre i dialoghi tra i personaggi, soprattutto se appartenenti a un ceto sociale basso, usano spesso la sintassi dialettale. Possiamo a tal proposito citare i numerosi casi di posposizione del verbo; inoltre l'uso del raddoppiamento del aggettivo con valore di superlativo assoluto (p. es. "Quando l'ondulante coda ciarliera scomparve dalla sua vista, mogio mogio se ne tornò a casa" ${ }^{17}$ ); la formazione del superlativo tramite l'aggiunta dell'avverbio "assai" in posizione finale ("Abbiamo un problema grosso assai"18); l'uso della preposizione "a" per introdurre l'accusativo, ovvero il complemento oggetto, nei casi in cui esso corrisponde a un essere animato ("Io a quella puttana non la servo"19), che ritroviamo anche in altri dialetti meridionali. A livello lessicale, Camilleri spiega il significato di parole siciliane poco comprensibili attraverso perifrasi e traduzioni. Per esempio, il verbo dialettale conzare significa in italiano "apparecchiare" ${ }^{20}$ Nel testo questa parola viene tradotta: "Allora, se non le dispiace, può conzarmi qui? Conzare, apparecchiare. Rizzitano disse quel verbo siciliano come uno straniero che si sforzasse di parlare la lingua del posto". ${ }^{21}$ Anche Agnello Hornby usa una strategia simile, inserendo le parole in un contesto esplicito, per far sì che il lettore che non ha familiarità con il dialetto siciliano possa intuire il loro significato. Nell'esempio che segue si veda il caso del verbo dialettale annacari, che in italiano vuol dire "cullare, dondolare, camminare dondolando i fianchi".22 "[...] 'faceva pure la mossa, con i pugni sui fianchi, e s'annacava tutta!' aggiunse Mario Lo Garbo alzandosi; imitò la mossa della Mennulara, mettendosi le mani sui fianchi e roteando goffamente il bacino sulle gambe magre e storte". ${ }^{23}$

Quando parliamo del livello lessicale, è importante tenere conto del fatto che i due racconti si svolgono in due periodi storici differenti. Il libro di Camilleri pubblicato nel 1996 racconta una storia contemporanea a quest'epoca. La storia della Agnello Hornby, pubblicata nel 2002, si svolge tra il 1908 e il 1963. Quindi i modi di esprimersi in questi due libri si differenziano tra di loro, cioè ognuno riflette le caratteristiche dell'epoca in cui la storia è ambientata. Nel libro della Agnello Hornby abbiamo alcuni tipi di saluto, che sono come afferma Leone: "andati

\footnotetext{
[15] Il cane di terracotta: 12

[16] Il cane di terracotta: 88

[17] La Mennulara: 67

[18] La Mennulara: 161

[19] La Mennulara: 105

[20] Siciliano-Italiano, 2012

[21] Il cane di terracotta: 263

[22] Siciliano-Italiano, 2012

[23] La Mennulara: 57
} 
cadendo dell'uso, spazzati via dalla ventata democratica degli ultimi decenni". ${ }^{24}$ Nel libro di Camilleri, che si colloca in un contesto storico più recente, non appare nessuna di queste forme di saluto.

Possiamo concludere che il libro di Camilleri presenta indubbiamente "un tasso" di dialettalità molto più alto rispetto al libro della Agnello Hornby. La dialettalità nel libro di Camilleri è ben presente a tutti i livelli, mentre nel libro della Agnello Hornby è più accentuata quella sintattica.

\section{[3] ANALISI DELLE TRADUZIONI NORVEGESI}

[3.1] Problemi circa la traduzione di varietà linguistiche non standardizzate: dialetti, idioletti e socioletti

Dinanzi a un testo che contiene varietà linguistiche non standardizzate, il traduttore deve scegliere se riportarle oppure non riprodurle nel testo d'arrivo, traducendo tutto il testo nella lingua nella quale si traduce. Federici (2011) distingue tra conservative e experimental approaches. Un approccio si dice conservativo quando si evita di sostituire dialetti, idioletti e socioletti con varietà non standardizzate della lingua di arrivo, e invece sperimentale quando si cerca di rivelare nella traduzione le differenze linguistiche presenti nel testo di partenza. ${ }^{25}$ Bellos (2011: 194), analizzando la prassi traduttiva nel caso delle varietà substandard, sottolinea che, se nel testo di partenza sono presenti dialetti, questi raramente verranno sostituiti con un idioma diatopicamente marcato nella lingua che si traduce:

"Most people currently think it is just silly to make a Bavarian dairy farmer use Texas cowboy slang, or to have a woman on the St. Petersburg tram express herself in Mancunian in order to suggest her geographic and linguistic distance both from the capital and the standard language".

Dunque la maggior parte delle traduzioni di questo tipo tende verso il centro, cioè verso il polo della lingua standard.

Bellos (2011) ricorda inoltre che per quanto riguarda i socioletti il comportamento dei traduttori è generalmente diverso rispetto a quello che riguarda $i$ dialetti. In questo caso si possono osservare due tendenze. Gli acroletti (lingua più elevata) vengono generalmente sostituiti con i loro corrispettivi nella lingua di arrivo: "High - flown, pompous, elegant, or regal forms of language in the source are

[24] Leone (1995: 23-24)

[25] "We can subdivide the types of rendering dialectal features of fictional narratives into conservative and experimental approaches. When translators do not attempt to force the norms, they are conservative in respecting the target language expectations and avoid challenging it with non- standard variants [...]. When translators try to reveal the differences in the source language, such as The Simpsons dubbed into Italian, which uses target language dialects ad absurdum [...], they are experimental" (Federici 2011: 10) 
generally represented by forms of corresponding social rank in the target" ${ }^{26}$ Però, i socioletti che rappresentano strati bassi della società spesso non sono sostituiti con corrispondenti basiletti della lingua d'arrivo. Questo perché i traduttori percepiscono il rischio che le loro scelte traduttive possano essere lette come i loro errori grammaticali o malapropismi. ${ }^{27}$

\section{[3.2] Le scelte traduttologiche di Jon Rognlien}

Che cosa è più importante nei romanzi di Camilleri, l'intreccio linguistico o la vicenda in sé? Il traduttore stesso racconta in un'intervista ne La Nota del Traduttore che nella sua traduzione la lingua di Camilleri non viene "restituita". La sua strategia comporta piuttosto "un tradimento radicale dell'idea di equivalen$\mathrm{za}^{28}$. Nella nostra intervista con Rognlien, alla domanda se lui fosse stato tentato dall'idea di usare un dialetto norvegese analogo a quello siciliano, ha risposto riportando come esempio il romanzo The colour purple di Alice Walker tradotto in norvegese con il titolo Fargen bortenfor. Una gran parte di questo romanzo, è scritta in un dialetto contadino usato dagli afroamericani negli Stati Uniti del sud. Il traduttore norvegese, Isak Rogde, ha scelto di sostituire questo idioma americano con il dialetto norvegese di Senja (a nord della Norvegia). Nella prefazione del libro, Rogde illustra il suo dilemma. Da un lato, se si sostituisce un dialetto straniero con un dialetto norvegese, c'è il rischio che il lettore possa fare delle associazioni che lo indirizzino verso un ambiente e un luogo che non corrispondono affatto all'ambiente e al luogo di provenienza. Però, dall'altro lato, se uno scrittore consapevolmente ha usato una forma linguistica che chiaramente si distingue da quella letteraria comune, quando nella traduzione questa non viene rispettata, c'è il rischio che una parte importante del racconto possa sparire. Rogde giustifica la sua scelta dicendo che per la scrittrice l'uso del dialetto è davvero importante e per questo egli non ha voluto eliminarlo, preferendo sostituirlo con quello di Senja che contiene alcuni tratti comuni al dialetto usato nel testo americano originale: entrambi sono fortemente caratterizzati dal distanziarsi dalla lingua ufficiale tanto al livello grammaticale che lessicale; entrambi appartengono a una comuni-

[26] Bellos (2011: 194-195)

[27] "Real difficulties arise only when the class register is low, and especially when the language of the source represents the speech forms of uneducated folk. This difficulty runs through all kinds of translating, not just literary prose. No consecutive interpreter, for example, would think of adopting lower- class diction to reproduce for the benefit of a visiting foreign dignitary the kind of language spoken to him by a factory hand or collective farm worker: it would surely seem disrespectful and cause a mighty scandal. In written prose too, translators shy away from giving the uncouth truly uncouth forms of language in the target text. The reason is obvious- grammatical mistakes, malapropisms, and other kinds of 'substandard' language must not be seen to be the translator's fault. [...] To put that a different way: translation always takes the register and level of naturally written prose up a notch or two. Some degree of raising is and always has been characteristic of translated texts- simply because translators are instinctively averse to the risk of being taken for less than fully cultivated writers of their target tongue" (Bellos 2011: 195)

[28] L'intera intervista si trova in: http://www. lanotadeltraduttore.it/domande_rognlien.htm 
tà marginalizzata e discriminata; entrambi non sono caratterizzati da snobismo, cioè mostrano genuinità e autenticità.

Ritornando a Rognlien, egli sottolinea che Rogde ha usato il dialetto di Senja perché è il suo dialetto d'origine e dunque ne ha una certa padronanza. Viceversa Rognlien, originario di Oslo, non ha la stessa competenza di un altro dialetto. Potrebbe optare per l'østnorsk, norvegese dall'est, dialetto che, però, non si distingue così tanto dal bokmål, almeno non come il siciliano si distingue dalla lingua standard italiana. Rognlien aggiunge che avrebbe potuto studiare un altro dialetto norvegese, fare delle ricerche, ma che tutto ciò non solo richiede molto tempo in partenza, ma è molto rischioso perché potrebbe alterare pesantemente il testo inducendo il lettore a fare delle associazioni che non corrispondono alla volontà dell'autore. Questo è un fatto importante: se si usa un dialetto norvegese molto marcato, questo ricorderà subito "il mare artico" e "il sole a mezzanotte" (le espressioni che usa Rognlien), ovvero allontanerà il lettore dal luogo in cui la storia in realtà si svolge. Tuttavia Rognlien sottolinea che con Camilleri il problema è più complicato. Il dialetto qui non è soltanto un elemento dal sapore pittoresco, è un elemento pienamente narrativo: "Camilleri usa il dialetto come un mattone in una costruzione narrativa" (espressione di Rognlien). Ogni personaggio non viene veramente descritto, nel senso tradizionale del termine, ma è attraverso il suo modo di parlare che il lettore impara a conoscerlo. Questo implica che l'uso del dialetto ha un significato particolare per la storia e che se questo viene sostituito, si perdono tutte quelle associazioni e quelle connotazioni che esso porta con sé. Rognlien sottolinea inoltre che ogni traduttore deve partire da zero, cioè considerare ogni volta quale significato il dialetto abbia per la storia che si deve tradurre e che cosa possa succedere se questo viene sostituito. Vari traduttori stranieri alle prese con Camilleri hanno risolto tale problema in svariati modi. Secondo Rognlien, la scelta di usare un dialetto solamente come se fosse un colore (ovvero giusto per inserire un elemento di variazione sociolinguistica nel testo) non tiene conto del suo ruolo funzionale nel testo. Nei libri di Camilleri, ad esempio, non figurano molte descrizioni del clima, del territorio o del cibo, poiché è appunto il dialetto siciliano con le sue immagini a coprire questo vuoto. Rognlien mette in evidenza che Camilleri, quindi, conta molto sulla competenza linguistico-culturale del lettore. La conoscenza dell'Italia e della sua cultura da parte di un lettore norvegese medio, non raggiunge tale livello. Rognlien lo spiega nell'intervista di Dori Agrosi:

“[...] per un norvegese qualunque l'Italia è prevalentemente una cosa [...], la competenza specifica del lettore qui in Norvegia è ristretta. E non scende al di sotto di un certo livello. [...] per riscuotere dal lettore della mia traduzione di Camilleri un giusto ripieno da mettere nei buchi del testo, ho scambiato il gioco 'dialettale' con un gioco 'nazionale', ragionando così: la distanza tra Firenze e Palermo si può in 
un certo senso paragonare alla distanza tra Norvegia e Italia. Ho scelto di lasciare parecchi richiami alla lingua italiana nel mio testo, usando parole che sono facilmente decifrabili (un po' come infatti è anche il siciliano di Camilleri - si capisce senza dizionario, almeno dopo un po') con titoli come 'commissario', 'avvocato', 'cavaliere', 'signora', nomi di piatti tipici, 'omertà', 'capo', certe locuzioni lasciate in corsivo e poi subito spiegate. In quel modo il testo cerca di fare un richiamo costante all'italianità del testo (che comprende la sicilianità, per noi). Il gioco è un altro, ma è analogo." 29

Peraltro egli nota che se ci fossero state più descrizioni, sarebbe stato un po' meno difficile sostituire il dialetto siciliano con un altro dialetto straniero, perché almeno attraverso di esse, la sensazione che la storia si svolga in Sicilia sarebbe stata più precisa. Giacché Camilleri risparmia sulle descrizioni e lascia alla lingua la maggior parte del compito di localizzare il racconto, diventa ancora più difficile sostituire il dialetto siciliano, perché questo porterebbe il lettore in tutt'altro posto.

Un'altra caratteristica importante che va tenuta in conto durante la traduzione è che la lingua di Camilleri è una specie di "ricamo linguistico" (come dice Rognlien) composto di vari livelli. Montalbano, per esempio, parla con una notevole ricchezza linguistica: sa parlare il siciliano stretto, ha una pardonanza perfetta dell'italiano e fa uso di una varietà battezzata "camilleriano". Quest'ultimo livello, che tanti credono essere siciliano, non lo è. Si tratta di una lingua creata da Camilleri stesso: "è una bell'illusione [...] lui mette un po' di lessico siciliano in una struttura italiana". ${ }^{30}$ Questa lingua raffinata, "il falso siciliano", ${ }^{31}$ composta da vari livelli è estremamente difficile da trasporre in norvegese.

Passando alle domande sulla prassi traduttiva in Norvegia riguardo l'utilizzo dei dialetti e dei socioletti, tema assai dibattuto nei translation studies, ${ }^{32}$ Rognlien descrive queste scelte come rischiose ("tetre" e "lunatiche") e spiega che quando un traduttore cerca di ricreare nella traduzione una varietà linguistica non standardizzata nella lingua d'arrivo, spesso questa viene percepita come fatta male, come se si trattasse di errori commessi dal traduttore, senza capire, invece, che è il testo originale ad essere scritto cosí. A questo proposito, Rognlien parla della traduzione norvegese del libro Balzac et la petite tailleuse chinoise di Dai Sijie, tradotto in norvegese da Bente Kristensen con il titolo Balzac og den lille kinesiske syersken. Questo romanzo è scritto in lingua francese, ma da un cinese, fatto evidente per un lettore francofono a causa della lingua usata. La traduttrice ha cercato di riportare questo particolare aspetto nella traduzione norvegese ed è stata fortemente

[29] L'intera intervista si trova in: http://www. lanotadeltraduttore.it/domande_rognlien.htm

[30] Intervista personale a J. Rognlien.

[31] ibid.

[32] Vedi per esempio Federici 2011. 
criticata per questo. Rognlien puntualizza, a tal proposito, che si tratta di un caso veramente difficile. Ne risulta una traduzione strana, perché il lettore non capisce che l'originale è scritto in una lingua che non è quella dell'autore.

Per quanto riguarda la traduzione norvegese The color purple Rognlien racconta che tanti sono dell'opinione che Rogde in questo caso è riuscito bene nell'intento. Nella prefazione del romanzo il traduttore giustifica le sue scelte. Questa è, secondo Rognlien, la via da intraprendere, contrariamente a B. Kristensen che non dà nessuna spiegazione al lettore. Così il lettore è preparato, e non percepisce le scelte traduttologiche come un errore del traduttore, ma è consapevole del fatto che il libro originale è scritto in un modo particolare.

Per concludere, Rognlien dice di aver fatto quello che ha potuto poiché si è trovato di fronte a un aspetto intraducibile dell'opera di Camilleri: il gioco linguistico è il vero protagonista, ciò che regge il racconto. Sarebbe stato poco opportuno usare i dialetti norvegesi, oppure le altre lingue scandinave, perché si sarebbero comunque perse le connotazioni culturali, altre immagini sarebbero state evocate, altre emozioni suscitate.

\section{[3.3] Analisi della traduzione "Terrakottahunden"}

La traduzione si apre con una prefazione nella quale il traduttore spiega la peculiarità della lingua camilleriana. Rognlien dice:

"Oversetteren kan velge om han vil prøve å lage en identisk maskin på sitt eget språk, i håp om at den vil oppføre seg noenlunde likt som originalen, eller han kan bygge en annen maskin som med litt hell og lykke frambringer et tilsvarende produkt." 33

Egli afferma di aver optato per l'ultima soluzione, cioè di non avvalersi dei dialetti, socioletti e gerghi norvegesi, bensì di tradurre il romanzo in bokmål, lasciando alcune parole italiane all'interno del testo.

Nella postfazione, Rognlien descrive in modo più approfondito la situazione sociolinguistica in Italia, le varietà della lingua italiana, e alcuni fenomeni culturali dell'Italia, e della Sicilia in particolare, che hanno dato vita a tante parole intraducibili in norvegese, quali sono le parole collegate alla mafia, ai gradi della polizia e alcune altre parole che descrivono la cultura materiale. Rognlien dedica anche un commento alla cucina siciliana: nel romanzo, infatti, sono nominati molti piatti tipici con ingredienti non familiari al lettore norvegese.

Le parole non tradotte in norvegese sono spesso nomi di professione come: il commissario, il questore, il vicequestore, dottore/dottori/dottor; nomi di piatti tipici

[33] "Il traduttore può scegliere se cercare di creare una macchina identica nella sua lingua, nella speranza che questa si comporti più o meno come nell'originale, o può costruire un'altra macchina che con un po' di fortuna produca un effetto corrispondente." (la traduzione è mia - P.T.) 
come tabisca, pasta 'ncasciata, le milanzane alla parmigiana; parole ed espressioni relative alla criminalità organizzata come pizzo, i capi-capi, omertà, pentito; parole ed espressioni relative alla storia italiana come i fasci di combattimento, il fascio littorio, giovane italiane e saluto romano; e alcune espressioni idiomatiche come vita, morte e miracoli. Il traduttore usa diverse strategie per tradurre o spiegare il significato: a volte aggiunge, con l'aiuto di perifrasi, una spiegazione o traduzione norvegese nel proprio testo (come vedremo di seguito), altre volte non lo fa.

Guardiamo il primo esempio, la prima volta che appare la parola carabinieri:

\section{(1) Il CANE DI TERRACOTTA}

"Lascia perdere. Telefona subito a Tortorella, digli che avverta Augello. Ci vadano loro due. Dicci che tu non ci puoi andare, contagli una minchiata qualsiasi, che sei caduto dalla culla e hai battuto la testa. Anzi, no: digli che i carabinieri sono venuti ad arrestarti. Meglio, telefona e digli d'avvertire l'Arma, tanto il fatto è cosa da niente, una cazzata di furto, e l'Arma diventa contenta perchél'abbiamo chiamata a collaborare" (p. 27).

\begin{abstract}
TERRAKOTTAHUNDEN
"Glem det. Ring til Tortorella med det samme og si at han skal varsle Augello. De to kan dra dit. Si at du ikke kan dra, fortell dem en hvilken som helst skrøne, at du har falt ut av vogga og slått hodet. Nei, forresten, fortell dem at carabinieri kom for å arrestere deg. Eller enda bedre, ring og si at de skal si fra til carabinieri, det er jo bare en fillesak, bare et jævla innbrudd, og da blir carabinieri fornøyd fordi vi har kontaktet dem for å samarbeide" (p. 24).
\end{abstract}

Rognlien lascia la parola italiana nella traduzione senza spiegarne il significato al lettore. Dal contesto si può capire che il termine carabinieri ha a che fare con la polizia. Vediamo anche che al posto della parola italiana l'arma, il traduttore usa la parola italiana carabinieri. È importante tenere conto del fatto che il corpo della polizia norvegese è diverso rispetto a quello italiano e spesso è difficile trovare un termine norvegese corrispondente. La parola carabinieri, insieme con i differenti gradi della polizia italiana, sono spiegati ulteriormente nella postfazione.

L'esempio seguente è una descrizione da parte della voce narrante in cui appare il nome del piatto milanzane alla parmigiana.

(2)

IL CANE DI TERRACOTTA

"Le milanzane alla parmigiana che la cammarera gli aveva lasciate nel forno gli parsero di colpo scipite, [...]" (p. 69).

\section{TERRAKOTTAHUNDEN}

"Den ovnsbakte aubergineretten - le melanzane alla parmigiana som hushjelpen hadde satt igjen til ham i ovnen, smakte plutselig ingenting, [...]" (p. 63). 
Vediamo che il traduttore ha lasciato il nome, ma non com'era stato scritto da Camilleri nell'originale; Rognlien scrive il nome del piatto in italiano standard: melanzane alla parmigiana e aggiunge una spiegazione su cosa il piatto sia: den ovnsbakte aubergineretten. ${ }^{34}$

Pizzo, nel senso "forma di tangente estorta dalle organizzazioni mafiose e camorristiche a negozianti, imprenditori ecc.", ${ }^{35}$ è un esempio di parola della quale non esiste il corrispondente norvegese. Questa è fortemente connotata $\mathrm{e}$ fa subito pensare alla mafia. Nel libro essa appare più volte e nella traduzione viene sempre lasciata in italiano. Ecco il passaggio in cui pizzo appare per la prima volta:

\section{(3) Il CANE DI TERRACOTTA}

"Persone alle quali non posso negarmi. Tu sai che io, come ogni commerciante, pago il pizzo per travagliare in santa pace e per non fare succedere burdello, fatto ad arte, nel burdello che ho. Ogni mese u Signuri Iddio manda in terra, c'è uno che passa e incassa" (p. 14).

\begin{abstract}
TERRAKOtTAHUNDEN
"Folk jeg ikke kan si nei til. Du vet at jeg, i likhet med alle andre som driver handel, betaler min pizzo, en andel av overskuddet for å kunne jobbe i ro og mak, slik at det ikke blir enda mer huskestue i den huskestuen jeg allerede bestyrer. Hver eneste måned Vårherre gir oss på jorden, kommer det noen og innkasserer" (p. 11).
\end{abstract}

Vediamo che subito dopo pizzo viene aggiunta una spiegazione "en andel av overskuddet". ${ }^{36}$ Il significato della parola viene spiegato ulteriormente nella postfazione.

\section{[3.4] Le scelte traduttologiche di Tommy Watz}

T. Watz nelle risposte alle nostre domande (date per iscritto) racconta che visto che l'uso del dialetto nel romanzo La Mennulara è piuttosto limitato, non si è posto la questione e non ha mai considerato la possibilità di usare un dialetto norvegese. Egli sostiene che, per quanto riguarda i tre libri della Agnello Hornby tradotti in norvegese da lui, La Mennulara, La zia marchese e La monaca, un lettore italiano medio ha pochi o nessun problema a capire le parole e le espressioni siciliane che vi si trovano che quindi non vengono percepite come ostacoli che bloccano o ritardano la lettura. Tradurre le rare espressioni dialettali in un qualsiasi dialetto norvegese renderebbe inutilmente più complessa la fruizione del testo tradotto rispetto a quello originale.

Watz sostiene che in tutti i libri italiani da lui tradotti, non ha mai usato altro che bokmål. Solo all'inizio della sua carriera, egli ha tradotto Racconti romani

[34] Letteralmente tradotto in italiano: la portata di melanzane fatte al forno.

[35] Voce presente nel dizionario Lo Zingarelli, 2011

[36] Letteralmente tradotto in italiano: una parte dell'eccedenza. 
di Alberto Moravia, con il titolo Italia forteller. In questo libro, accanto all'italiano, viene usato anche il dialetto romanesco. Watz ha provato a ricreare questo effetto di diglossia, traducendo in un dialetto, che lui in quel periodo considerava il suo dialetto, ovvero la lingua che si parla nella zona orientale di Oslo. Il risultato però, secondo lui, non può considerarsi ben riuscito, anche per il fatto che si è reso conto di non padroneggiare più perfettamente il suo dialetto d'origine. Magnus Ulleland, filologo norvegese, traduttore dei classici italiani nonché rilettore molto autorevole dell'Italia forteller, ha dato un giudizio fortemente critico su questa operazione, inducendo Watz a desistere dalla sua scelta. Come Rognlien, Watz afferma di conoscere solamente l'østnorsk, e le poche varianti di questa forma dialettale, perché nato e cresciuto a Oslo. Se si fosse reso necessario l'utilizzo di un altro dialetto in una delle sue traduzioni, avrebbe avuto bisogno di un aiuto esterno; senza contare che sarebbe stato un modo di lavorare estraneo per lui. Watz continua dicendo di non aver mai letto una traduzione norvegese in cui l'utilizzo di dialetto abbia funzionato bene. Come Rognlien, egli ricorda l'esempio della traduzione norvegese del romanzo di Alice Walker The color purple e menziona anche un'altra traduzione norvegese di un libro di Takiji Kobayashi, tradotto da Magne Tørrings con il titolo Krabbeskipet. In questa traduzione, il traduttore norvegese fa parlare gli uomini dell'equipaggio di una nave da pesca giapponese con un dialetto del Nord-Troms. Watz sostiene che entrambi questi esempi sono dei tentativi falliti. L'introduzione di un dialetto fa sì che i libri diventino pesanti da leggere per un lettore a cui non è familiare il dialetto scelto.

Watz dice che, secondo le sue esperienze, i lettori norvegesi non conoscono l'Italia e la sua cultura. La maggior parte dei libri che vengono tradotti dall'italiano appaiono come abbastanza esotici già dal principio e non hanno bisogno di un ulteriore "straniamento" che potrebbe derivare da scelte lessicali ingiustificate. Nomi di luoghi, di titoli, di professioni, di piatti e altri elementi culturali sono già abbastanza carichi di un certo colorito locale. Coerentemente con questa posizione teorica, Watz menziona la sua traduzione del libro della Agnello Hornby, La zia marchese. In questo libro, infatti, ogni capitolo è introdotto da un proverbio siciliano. Ci sono 84 capitoli e dunque 84 proverbi in dialetto. In questo caso, Watz ha scelto di lasciare i proverbi in siciliano per poi tradurli più o meno direttamente in norvegese, invece di cercare di trovare 84 corrispondenti proverbi norvegesi. A proposito della traduzione norvegese del libro di Marco Malvaldi La carta più alta, tradotto con il titolo Det høyeste kortet, Watz racconta che qui figura un dialetto della zona vicino a Pisa. Per di più la lingua è molto volgare. Quasi ogni due frasi appaiono parole scurrili come cazzo, culo, coglioni. In questo caso, Watz non ha sostituito il dialetto pisano con un dialetto norvegese, ma ha fatto parlare i personaggi in un modo popolare permettendo loro di usare espressioni molto volgari. Watz dice: 
"Jeg ville finne det høyst unaturlig å utstyre Malvaldis kortspillende pensjonister med en dialekt som leder tankene mer hen til tørrfisk og rorbu enn til Toscana- kystens pinjelunder, selv om frisk bannskap etter sigende skal være mer utbredt nord for Trondheimsfjorden". ${ }^{37}$

Per concludere, va notato che Watz si è trovato di fronte a un problema diverso da quello di Rognlien. Visto che nel romanzo di Agnello Hornby, la funzione del dialetto non ha un'importanza pari a quella che riveste in Camilleri, Watz ha avuto ragione a non sottolinearne la presenza. Nella sua traduzione, la mancanza di piccole sfumature dialettali non è tutto sommato una grande perdita, giacché la storia regge bene anche senza di esse.

\section{[3.5] Analisi della traduzione "Mandelplukkersken"}

La traduzione di Watz non ha una prefazione né una postfazione. Il libro è stato tradotto in bokmål e le poche sfumature dialettali presenti nell'originale non sono state tradotte; il traduttore non ha provato a riprodurre i vari registri linguistici, tranne nei rarissimi casi che verranno commentati in seguito.

Vediamo un esempio con la parola dialettale cosca che significa "ciascun piccolo nucleo di malfattori affiliato alla mafia siciliana". ${ }^{38}$ Nel libro la parola appare una volta:

\section{(4) La MEnNulara}

"[...], che addirittura parla di cosche mafiose, traffico di droga, armi, insomma farnetica, [...]" (p. 159).

\begin{abstract}
MANDELPLUKKERSKEN
“[...] og han snakker sågar om bandekrig, om narkotikatrafikk og våpensmugling, kort sagt han snakker over seg, [...]" (p. 204).
\end{abstract}

Vediamo che nel testo di partenza dopo la parola cosche segue l'aggettivo "mafiose", nel testo d'arrivo però, cosche è stato tradotto con bandekrig ${ }^{39}$ e l'aggettivo "mafiose" è stato tolto. Dal contesto, il lettore norvegese percepisce che si tratta di una banda criminale, ma che sia proprio la mafia potrebbe essere poco chiaro.

In tutto il testo di partenza appaiono due espressioni idiomatiche siciliane. Queste vengono sempre tradotte in norvegese. Guardiamo le scelte traduttologiche di queste due espressioni: lu piru fa pira.

[37] "Avrei trovato estremamente innaturale affibbiare ai giocatori di carte pensionati di Malvaldi un dialetto che avrebbe indotto a pensare più a stoccafissi e capanne di pescatori che alle pinete della costa toscana, malgrado l'uso di bestemmiare in modo colorito pare che sia alquanto più diffuso a nord del fiordo di Trondheim".

[38] Voce presente nel dizionario Lo Zingarelli, 2011.

[39] Letteralmente tradotto in italiano: guerra di banda. 
(5) La Mennulara

"Senza tradire il segreto della confessione, questo solo vi dico: non mi sorprende quanto mi raccontate dell'avvocato Orazio... come dice il proverbio, 'lu piru fa pira', il pero produce pere, e di femmine di quel tipo, introdotte di nascosto nelle case private, di notte e di giorno, ce ne sono tante..." (p.155).
MANDELPLUKKERSKEN

"Uten å bryte skriftemålets taushetsplikt kan jeg si Dem at det De forteller meg om Orazio, ikke forundrer meg... Som det heter i ordtaket: 'Eplet faller ikke langt fra stammen', og mange kvinner av den sorten er, i ly av nattens mulm og mørke eller sågar på høylys dag, blitt smuglet in i private hjem..." (p. 198).

Nel testo di partenza dopo l'espressione viene scritto in italiano il "pero produce pere": il proverbio siciliano corrisponde al proverbio italiano "la mela non cade mai troppo lontana dall'albero". Watz ha tradotto con l'espressione idiomatica: eplet faller ikke langt fra stammen, ${ }^{40}$ che corrisponde perfettamente al senso dell'espressione idiomatica siciliana, ma non alla sua forma dialettale. Vediamo che nel testo originale il proverbio viene scritto prima in siciliano, per poi essere tradotto in italiano, ma nel testo d'arrivo Watz scrive il proverbio solamente in norvegese.

Guardiamo l'espressione: fimmina di panza, che si traduce letteralmente come "femmina di pancia". In siciliano esiste un'espressione idiomatica o'mini i pansa (uomo di pancia) il cui senso è: "Un uomo che sa trattenere nella sua pancia ogni segreto; quindi uomo che non parla, che pratica l'omertà, mafioso". ${ }^{41}$ Sembra che la Agnello Hornby abbia trasposto il significato di o'mini i pansa in fimmina di pan$z a$. Quest'espressione appare tre volte nel libro; vediamo come Watz ha scelto di tradurla nei vari casi:

(6)

La Mennulara

"Fatemi la cortesia di andare a riferire a don Vincenzo Ancona che la Mennulara gli manda i suoi saluti e che lo chiamerò presto, che non si scomodi a contattarmi, gli manderò una voce quando sono pronta a parlagli, e che non si preoccupi, 'fimmina di panza sono io', e ci rimango" (p. 69).

\section{MANDELPLUKKERSKEN}

“Gjør meg derfor den tjeneste å hilse don Vincenzo Ancona så mye fra meg, Mennulara, og si at jeg tar kontakt med ham så snart jeg kan, at han ikke behøver å ta seg det bryderi å kontakte meg, men at jeg gir ham beskjed så snart det passer meg å snakke med ham, og at han ikke har noe grunn til å bekymring for jeg er et 'ordholdent kvinnfolk', og det vil jeg fortsette å være også i fremtiden" (p. 90).

[40] Letteralmente tradotto in italiano: la mela non cade lontano dal tronco.

[41] Voce presente nel Dizionario Siciliano- Italiano, 2012. 
Nel primo caso Watz ha tradotto l'espressione con ordholdent kvinnfolk. ${ }^{42}$ In questo modo si mantiene il senso dell'espressione siciliana, ma è chiaro che le connotazioni legate alla mafia si perdono.

(7) La Mennulara

"A casa si rideva della sua segretezza: mio padre, che era nell'arma dei Carabinieri, sosteneva che se fosse nata maschio sarebbe diventata capomafia, la chiamava 'fimmina di panza'." (p. 135).

\begin{abstract}
MANDELPLUKKERSKEN
"Hjemme lo de av alt hemmelighetskremmeriet hennes; min far, som var i carabinierikorpset, hevdet at dersom hun var blitt født mann, ville hun ha vært mafiaboss, han kalte henne en 'kvinne av ære'." (p. 176).
\end{abstract}

Nel secondo caso, per contro, vediamo che l'espressione è stata tradotta diversamente, cioè in kvinne av cere..$^{43}$ Come sopra, se Watz, da un lato, riesce a trasporre il senso dell'espressione, dall'altro, però, è costretto a perdere dei tratti culturospecifici (il mondo della malavita), che però sono recuperabili dal contesto.

(8) La Mennulara

"Insomma, aveva la bocca cucita come una "fimmina di panza"' (p. 196).

\section{MANDELPLUKKERSKEN}

"Kort sagt, hennes munn var lukket med syv segl; hun var en kvinne til å stole på” (p. 249).

L'ultima volta che appare la stessa espressione, Watz sceglie di nuovo di sostituirla con hennes munn var lukket med syv segl, ${ }^{44}$ espressione che identifica una persona capace di mantenere tutti i segreti, dopodiché aggiunge: hun var en kvinne til å stole $p a^{45}{ }^{45}$ probabilmente perché è un'espressione che potrebbe risultare antiquata al lettore norvegese più giovane. Watz è riuscito a riportare in norvegese il senso di questo modo di dire, ma chiaramente le associazioni alla mafia non potrebbero mai essere tradotte in norvegese con parole o espressioni equivalenti.

Nonostante l'approccio di Watz sia quello di non lasciare tracce della lingua italiana o del dialetto siciliano nella sua traduzione, ci sono tuttavia alcune parole che sono lasciate nella loro forma originale, e sono zia/zu', don e donna, quando accompagnano il nome proprio. Si osservi l'esempio seguente:

[42] Letteralmente tradotto in italiano: una donna di parola.

[43] Letteralmente tradotto in italiano: donna d'onore.

[44] Letteralmente tradotto in italiano: la sua bocca era chiusa con sette sigilli.

[45] Letteralmente tradotto in italiano: era una donna della quale ci si poteva fidare. 
(9) La Mennulara

"Zia Carmelina Li Pira, la zia nubile di donna Enza e donna Mimma, anziana e alquanto svanita, era stata presa in casa dai Militello" (p. 31).
MANDELPLUKKERSKEN

"Zia Carmelina Li Pira, donna Enzas og donna Mimmas ugifte tante, gammel og temmelig tussete, bodde hos ekteparet Militello" (p. 41).

In questo esempio la parola zia appare per la prima volta, ma non viene tradotta. Invece la seconda volta che appare, viene tradotta con il corrispettivo norvegesetante. Il lettore norvegese che non conosce la lingua italiana probabilmente non percepisce che zia sia una parola italiana pensando che faccia parte del nome proprio. Come mai Watz contravviene in questo punto i suoi stessi principi traduttivi?

Watz giustifica la sua scelta dicendo che non sarebbe giusto tradurre letteralmente le parole "zia/zio", perché in questo romanzo, esse non devono essere sempre interpretate nel loro significato proprio: molto spesso si tratta di una forma appellativa di rispetto usata nel Sud per rivolgersi a persone anziane. Per esempio, nello stesso romanzo appare anche una figura chiamata "Zu' Peppino", ma non è lo zio di nessuno. Watz sottolinea che in norvegese non esiste una forma appellativa corrispondente che potrebbe funzionare nella traduzione, e quindi la sua scelta è stata quella di lasciare le forme in italiano. Durante la sua carriera come traduttore, con circa settanta traduzioni all'attivo, egli ha cercato di introdurre questa forma per renderla familiare al pubblico norvegese. La prima volta che incontrò questo uso di "zia" fu all'inizio degli anni Novanta traducendo il dramma di Lina Wertmüller "Amore e magia nella cucina di mamma", dove la figura centrale veniva chiamata "Zi' Tripolina" sia nella versione norvegese bokmål che in nynorsk. E da allora Watz si comporta in modo lineare, mantenendo sempre la forma appellativa.

Il traduttore usa la stessa strategia per gli appellativi "Don/Donna" e argomenta che, grazie a grandi opere come La Cenerentola e Don Giovanni, queste forme sono ormai familiari per il pubblico norvegese. Anche in questo caso, non sarebbe sempre giusto tradurre in norvegese "Don/Donna" con Herr/Fru, perché si tratta di una forma appellativa di rispetto con connotazioni diverse. Per esempio, spesso anche nella letteratura mafiosa si utilizzano queste espressioni relativamente ai capi mafiosi, com'è il caso del romanzo, oggetto della nostra analisi, La Mennulara.

Watz continua dicendo che nel caso dell'appellativo "Signor(e)/Signora" si comporta diversamente: non lascia queste forme in italiano, ma le traduce in "Herr/Fru". È un argomento che Watz ha discusso più volte con gli altri traduttori in diverse lingue, e su cui tutti i traduttori sono d'accordo. Infatti, "Signore/Signora" viene tradotto in inglese con "Mr/Mrs", in francese, per la maggior parte, con "Monsieur/Madame", e in spagnolo con "Señor/Señora". Questa scelta 
che potrebbe anche sembrare discutibile, secondo Watz, è di solito seguita nella maggior parte delle traduzioni.

\section{[4] CONCLUSIONE}

Confrontati gli approcci di Rognlien e di Watz, possiamo riassumere dicendo che l'approccio del primo traduttore è piuttosto sperimentale (experimental nei termini di Federici 2011), mentre l'approccio del secondo è decisamente più conservatore (conservative). Tuttavia, è importante tenere presente che il ruolo del dialetto nei due romanzi analizzati è diverso; quindi le considerazioni fatte e le strategie scelte dai due traduttori non possono che essere differenti. Dato che il gioco linguistico di Camilleri è nodale per la storia e, per dirlo con le parole di Rognlien, costituisce "un elemento narrativo"; abbiamo visto che il traduttore ha scelto di fare un gioco analogo a quello di Camilleri, e ci è riuscito per quanto possibile. Invece nel libro della Agnello Hornby, il dialetto non ha un ruolo così fondamentale. Del resto, la scrittrice stessa afferma nella nostra intervista che il fatto di non tradurre, o meglio di sostituire il dialetto, non comporta una grande perdita. Non a caso, Watz ricorda di non aver nemmeno considerato l'alternativa di usare un dialetto norvegese, proprio per il fatto che il dialetto è quasi assente dal testo di partenza. Watz ha tradotto tutto il testo in bokmål (tranne le parole $z u^{\prime}$, don e donna), senza far notare al lettore norvegese che nel testo originale a volte era presente una forma di code switching. Non ci sentiamo di criticare questa scelta perché, come abbiamo detto, si tratta di una piccola perdita che non altera il racconto. Ad ogni modo, dalle due interviste fatte ai traduttori, possiamo constatare come le loro idee sono in generale alquanto simili. Innanzitutto, nessun dialetto norvegese può creare le stesse associazioni dei dialetti italiani. Riguardo la traduzione di La carta più alta di Malvaldi, Watz si trova d'accordo con Rognlien sul fatto che un dialetto norvegese porterebbe il lettore nel posto sbagliato. Dunque sembra chiaro che entrambi i traduttori condividano la stessa opinione: se si sceglie di sostituire un dialetto straniero con un dialetto norvegese, c'è il rischio che il lettore riceva delle associazioni linguistiche che deraglierebbero in direzione di un ambiente e di un luogo che non corrispondono affatto a quelli da cui provengono i personaggi della storia. Watz afferma di non aver mai visto una traduzione ben riuscita, in cui un traduttore ha sostituito un dialetto straniero con un dialetto norvegese, e che tutti i casi del genere sono, secondo lui, tentativi falliti. Rognlien dice che è una cosa rischiosa portare una forma di varietà linguistica non standardizzata in una traduzione, e spesso il risultato viene percepito come una traduzione fatta male.

Attraverso la riflessione sui problemi di traduzione di un libro scritto in lingua standard insieme con varietà linguistiche non standardizzate, abbiamo visto che questa tematica è sempre stata ed è ancora oggi molto discussa sia da linguisti sia da traduttori e che una risposta univoca su come risolvere questo problema 
non esiste. Per il futuro sarebbe interessante analizzare l'uso del dialetto in altri scrittori novecenteschi, ad esempio Sciascia e Pirandello, e le strategie dei loro traduttori. Questo permetterebbe di vedere come cambia l'approccio alla presenza di una o più varietà linguistiche non standardizzate nel testo di partenza e nel testo di arrivo.

\section{RIF ERIMEN T I B I B L I O GR A F I C I}

Agnello Hornby, S. 2005. Mandelplukkersken. Trad. da T. Watz. Oslo: Pax forlag. Agnello Hornby, S. 2010. La Mennulara. $15^{\text {a }}$ ed. Milano: Feltrinelli.

Auer, P. 2005. Europe's sociolinguistic unity, or: A typology of European dialect / standard constellations. In N. Delbecque, J.V.D. Auwera \& D. Geeraerts (cur.), Perspectives on Variation: Sociolinguistics, Historical, Comparative, 7-42. Berlin: Mouton De Gruyter.

Bellos, D. 2011. Is that a fish in your ear? Translation and the meaning of everything. New York: Faber and Faber.

Berruto, G. 2012. Sociolinguistica dell'italiano contemporaneo. $2^{\mathrm{a}}$ ed. Roma: Carocci. Brevini, F. 2010. La letteratura degli italiani. Perché molti la celebrano e pochi la amano. Milano: Feltrinelli.

Buratti, R. 2009. Italiano. Verbi. Milano: Vallardi.

Camilleri, A. 2005. Terrakottahunden. Trad. da Rognlien. Oslo: Gyldendal.

Camilleri, A. 2011. Il cane di terracotta. $55^{\mathrm{a}}$ ed. Palermo: Sellerio.

Cerrato, M. 2012. L'alzata d'ingegno. Analisi sociolinguistica dei romanzi di Andrea Camilleri. Firenze: Franco Cesati.

De Renzo, F. 2008. Per un'analisi della situazione sociolinguistica dell'Italia contemporanea. Italiano, dialetti e altre lingue. Italica 85(1). 44-62.

Eco, U. 2007. Dire quasi la stessa cosa. Esperienze di traduzione. $7^{\mathrm{a}}$ ed. Milano: Bompiani.

Federici, F. 2011. Introduction: Dialects, idiolects, sociolects: Translation problems or creative stimuli? In F. Federici (cur.), Translating Dialects and Languages of Minorities. Challenges and Solutions. 1-20. Bern: Peter Lang.

Grassi, C., A.A. Sobrero \& T. Telmon. 2012. Fondamenti di dialettologia italiana. RomaBari: Editori Laterza.

Gutkowski, E. 2009. Does the Night Smell the Same in Italy and in English Speaking Countries? An essay on translation: Camilleri in English. Enna: IlionBooks.

Leone, A. 1995. Profilo di Sintassi siciliana. Palermo: Atlante Linguistico della Sicilia. Lorenzetti, L. 2002. L'italiano contemporaneo. Roma: Carocci.

Marazzini, C. 2004. Breve storia della lingua italiana. Bologna: Il Mulino.

Marcato, C. 2002. Dialetto, dialetti e italiano. Bologna: Il Mulino.

Nergaard, S. (cur.). 2009. La teoria della traduzione nella storia. $4^{\mathrm{a}}$ ed. Milano: Gruppo editoriale Fabbri-Bompiani-Sonzogno-ETAS.

Pitrè, G. 2008. Grammatica siciliana. Palermo: Sellerio. 
Salvi, G. \& Vanelli L. 2004. Nuova grammatica italiana. Bologna: Il Mulino.

Schleiermacher, F. 2009. Sui diversi metodi del tradurre. In S. Nergaard (cur.), La teoria della traduzione nella storia, $4^{\mathrm{a}}$ ed., 143-179. Milano: Gruppo editoriale Fabbri-Bompiani-Sonzogno-ETAS.

Skjekkeland, M. 2010. Dialektlandet. Kristiansand: Portal forlag.

Sobrero, A. A. (cur.). 2011. Introduzione all'italiano contemporaneo. $11^{\mathrm{a}} \mathrm{ed}$. Roma-Bari: Editori Laterza.

Sorgi, M. 2000. La testa ci fa dire. Dialogo con Andrea Camilleri. Palermo: Sellerio editore Palermo.

Tropea, G. 1976. Italiano di Sicilia. Palermo: Aracne.

\section{DIZIONAR I}

Bonfiglio, G. 2012. Siciliano-Italiano: Piccolo vocabolario ad uso e consumo dei lettori di Camilleri e dei siciliani di mare. $2^{\mathrm{a}}$ ed. Roma: Fermento.

Italiensk blå ordbok, italiensk-norsk / norsk-italiensk. 2002. Oslo: Kunnskapsforlaget. Lo Zingarelli, $12^{\mathrm{a}}$ ed. 2011. Vocabolario della lingua italiana. Bologna: Zanichelli.

Scavuzzo, C. 1982. Dizionario del parlar siciliano. Palermo: Edikronos.

Sorge, P. 2011. Dizionario dei modi di dire della lingua italiana. $3^{\mathrm{a}}$ ed. Roma: Newton Compton Editori.

Vocabolario siciliano-italiano. 2001. Catania: Brancato Editore.

\section{S I T O G R A F I A}

Accademia Apulia UK. Simonetta Agnello Hornby avvocato-scrittrice. 2013. http : / / www . accademiapulia . org/it/members / simonetta - agnellohornby avvocato-scrittrice-244.html (20 febbraio, 2013). online.

Camilleri fans club. Il dialetto nei romanzi di Andrea Camilleri. 2013. http : / / www . vigata.org/dialetto_camilleri/dialetto_camilleri.shtml (8 febbraio, 2013). online.

Feltrinelli. Biografia. 2013. http://www . agnellohornby. it/biografia/ (20 febbraio, 2013). online.

La Nota del Traduttore. 10 domande a Jon Rognlien, traduttore di Camilleri in norvegese. 2013. http : / / www . lanotadeltraduttore.it/domande_rognlien.htm (4 marzo, 2013). online.

RaiLibro. L'investigatore e Camilleri.2013. http://www.railibro.rai.it/interviste . asp?id=210 (8 febbraio, 2013). online.

Scrittorincorso. Linguaggio. 2013. http://www . andreacamilleri.net/camilleri/ linguaggio. $\mathrm{html}$ (8 febbraio, 2013). online.

Språkrådet. Fakta om norsk språk. 2013. http : / / www . sprakrad . no/PolitikkFakta/Fakta/ (24 giugno, 2013). online. 
AUTHOR CONTACT INFORMATION

Pernille Thull

Università di Oslo

Sapienza Università di Roma

p_thull@hotmail.com 